\title{
Introduction to User Experience (UX) In Information Systems (IS) for Health and Wellness Minitrack - HICSS 50-2017
}

\author{
Ann Fruhling \\ University of Nebraska - Omaha \\ afruhling@unomaha.edu
}

\author{
Rich Burkhard \\ San Jose State University \\ richard.burkhard@sjsu.edu
}

\author{
Soussan Djamasbi \\ Worcester Polytechnic Institute \\ djamasbi@wpi.edu
}

Smartphones and a vast array of new wearable devices are empowering patients and healthcare professionals, bringing a fundamental shift in the health monitoring and decision making process and putting the consumers (patients) in charge. Today's consumers are less impressed by basic usability and functionality, and they seek compelling user experiences. Demand for positive user experiences creates a need for innovation in user experience approaches for health and wellness products and services. Examination of healthcare IT systems through an UX lens provides opportunities for both HCI and consumer health informatics researchers to formulate new theories and practices, and develop unique, new technologies for health and wellness.

This year, the Minitrack team selected six excellent articles from a large pool of highly competitive submissions.

Our first presentation is "Simplifying Fitness Games for Users with Learning Disabilities" by Liu Liu, Patrick Stacey, Monideepa Tarafdar, and Nikolaos Kourentzes. This research examines the challenge of motivating people with learning disabilities to perform physical exercises and the need for fitness games that are suitable for users with learning disabilities. The authors surveyed 235 people from both gaming and healthcare industries, leading to proposed features of fitness games for this user group.

Next, "Defining Dimensions of Patient Satisfaction with Telemedicine: An Analysis of Existing Measurement Instruments," is presented by Robert Garcia, Adelakun Olayele, and Wencui Han. This study applies a grounded theory approach to analyze patient satisfaction instruments, leading to several measurement approaches for describing patient satisfaction with telemedicine. The author's logic makes use of 22 unique constructs in this effort.

Our third article is "'I don't bother with the phone!': Feeling Closer to Physician using Secure
Messaging," by Yunan Chen and Charlotte Tang. The authors explore the use of phone versus secure messaging in online patient portals in the communication between patients and their healthcare providers. Patients appear to often prefer secure messaging in this context, which suggests another shift in patient-provider communication.

Next, authors Mahmoud Heshmat, Jaehyun Park, and Noha Mostafa present "Towards Patient-oriented Design: A Case of the Egyptian Private Outpatient Clinics." This case study examined patient involvement in private clinics in Egypt, which led to a design for a patient service solution. The design was prototyped in the authors" "YourClinic" online booking and consultation application service.

Our fifth presentation is "Effective Use of User Interface and User Experience in an mHealth Application," by researchers Wonchan Choi and Bengisu Tulu. The authors apply Effective Use Theory to examine the effect of UI design on effective use in a world of mobile applications. The researchers tested four mobile applications using a controlled experiment and a follow-up survey. Key findings have clear implications for interface design for effective use.

Next, authors Kuniomi Shibata, Akira Hattori and Sayaka Matsumoto present “'Media for Capability' of Children with Disabilities: Development of the Japanese Augmentative Communication App by Tablet for Persons with Hard of Hearing," which examines the potential role of information technologies to assist hearing-impaired persons in Japan. The authors discuss a unique Android-based application for expressing texts and images for children with hearing challenges.

We are honored by the continued strong interest in the User Experience in Information Systems for Health and Wellness Minitrack and would like to thank all seventeen of this year's contributing authors for submitting their outstanding research. 